\title{
Affect and Social Processes in Online Communication - Experiments with an Affective Dialog System
}

\author{
Marcin Skowron, Mathias Theunis, Stefan Rank, and Arvid Kappas
}

\begin{abstract}
This paper presents an integrated view on a series of experiments conducted with an affective dialog system, applied as a tool in studies of emotions and social processes in online communication. The different realizations of the system are evaluated in three experimental setups in order to verify effects of affective profiles, as well as of fine-grained communication scenarios on users' expressions of affective states, experienced emotional changes, and interaction patterns. Results demonstrate that the system applied in virtual reality settings matches a Wizard-of-Oz in terms of chatting enjoyment, dialog coherence and realism. Variants of the system's affective profile significantly influence the rating of chatting enjoyment and an emotional connection. Self-reported emotional changes experienced by participants during an interaction with the system are in line with the type of applied profile. Analysis of interaction patterns, i.e., usage of particular dialog act classes, word categories, and textual expressions of affective states for different scenarios, demonstrates that a communication scenario for social sharing of emotions was successfully established. The experimental evidence provides valuable input for applications of affective dialog systems and strengthens them as valid tools for studying affect and social aspects in online communication.
\end{abstract}

Index Terms-Affective dialog system, human-computer interaction, affect sensing and analysis, structuring affective interactions.

\section{INTRODUCTION}

The study of collective emotions on the Internet provides rich evidence of their influence in the process of creation, formation and breaking up of online communities. The growing impact of online communities that often form spontaneously and in which emotional factors play a crucial role is widely acknowledged [1]. The last years provided numerous examples of their importance in the fields of politics, sports, entertainment, culture and economics. Furthermore, there appears to be a tight coupling between collective emotions expressed on the Internet and their impact on the events reaching far beyond the online world. Recent studies are primarily focused on the analysis and modeling of relations between orientation and intensity of valence exchanged in textual messages and the interaction patterns in ecommunities [2], [3], [4], [5], [6], as well as physiological responses related to the perception and generation of emotionally charged online content [7], [8]. The method presented here complements these lines of work, and employs an affective dialog system to provide experimental setups for studying the impact of a range of affective and social factors in online communication with Internet users. It is targeted to apply networked computers and the Internet in particular for the exploration of emotion,

- M. Skowron and S. Rank are with the Austrian Institute for Artificial Intelligence (OFAI), Vienna, Austria.

E-mail: marcin.skowron / stefan.rank @ ofai.at

- M. Theunis and A. Kappas are with the School of Humanities and Social Sciences, Jacobs University, Bremen, Germany.

E-mail: m.theunis / a.kappas@ jacobs-university.de self and sociability [9], [10]. The presented affective dialog system extends this notion: (i) by providing specific experimental setups which support studies on how interactions with machines influence us, (ii) by simulating human participants - in a specific interaction setting - which contributes to studies on human-human communication processes, in particular in mediated and virtual online settings, (iii) by enabling the realization of interaction scenarios in which differences between human and artificial communication partners can be investigated. In our approach, the interdisciplinary collaboration between psychologists and computer scientists leads to mutual benefits to both disciplines. Studies in human interaction from psychology and communication inform the design of dialog systems on the one hand. On the other hand, the possibility to determine particular aspects of system behaviors allows new insights for psychologists on human responses to particular behaviors.

The focus of the research reported here is on development and evaluation of the affective dialog system. Specifically, it relates to the areas of affect detection [11] and affect modeling and generation [12], [13], with a specific emphasis on their application in interactive systems using predominantly a textual modality. In the case of the presented experiments, this means unrestricted, natural language based online conversations between system and participants. The main goals of the studies described below aim thus at investigating the methods useful for the creation and consistent simulation of affective profiles, personalities and fine-grained communication scenarios in such systems. The core research questions of the studies focus on the effects of the sim- 
ulated affective and interactive behavior of an artificial system on the participants. Specifically, we investigate the influence of structuring of emotional interactions on the communication style of users, textual expressions of affect, self-reported emotional states, and impressions regarding a conversational partner. Further, we analyze how those components relate to each other and account for the changes introduced artificially or occurring naturally during the course of online interactions. This includes the application of sentiment mining and affect analysis tools and resources, and their integration with dialog management and generation components.

On a macro-scale, the presented system serves as a tool, enabling extended analyses of collective emotions in a quantitative manner, e.g., by reaching out to groups of users that do not voice their opinions on other communication channels, and in qualitative ways, e.g., by conducting follow-up dialogs related to topics of interest, either introduced by the system or naturally occurring during an interaction. On a micro-scale, the focus of this paper, the presented system is applied for studying the role of emotions and social processes in affective HCI. With this line of research, we create autonomous systems usable in an experimental setup for investigating effects of changes in the affective or social dimensions of communication patterns and interaction scenarios. The fundamental assumption at the psychological level is linked to a basic tenet of appraisal theories, namely that personal relevance is associated with increased affective responses ${ }^{1}$.

This paper presents an integrated view on a series of recent experiments conducted with an affective dialog system, the Affect Listener. First, the dialog system is evaluated in a virtual reality (VR) environment in comparison with a Wizard-of-Oz (WOZ) setting in terms of dialog realism, dialog coherence, and participants' feeling of an emotional connection with their conversational partner. Second, the system is applied in a setting typical for online, real-time, text-based communication, e.g., chat-rooms, as a tool for studying the effect of affective profiles on communication patterns, expressions of affective states and self-reported emotional changes experienced by participants. Third, the system is used for studying the effect of fine-grained communication scenarios, "sharing of an emotionally intense personal episode" and "getting acquainted with someone", on the participants and their communication patterns and expressions of affective states.

The first two studies were originally described in [14], [15], [16]. This paper extends the previous contributions by: providing more detailed analysis of the experimental

1. We use appraisal theories as reference for the elicitation mechanisms of emotions. The central representation of emotional states in the system is dimensional, based on valence, arousal, and, to a smaller extent, dominance. This choice is based on what text analysis tools can provide and what physiological measurements in psychological studies relate to. This dimensional representation was also chosen as part of the overarching framework for the EU project CYBEREMOTIONS that supported this work. data, introducing the new experimental setup and results obtained in the third round of experiments, presenting new analysis of the system's communication patterns and its affective dimensions - for all three rounds of experiments, and offering a comparison between the three studies and analysis of the data-sets.

The next section covers the relation to relevant research. Sections 3 and 4 introduce the method applied in the studies, i.e., an overview of the affective dialog system, and describe the interaction scenarios used in the three rounds of experiments. Section 5 presents the experimental results. Section 6 reports the general findings from the three rounds of experiments. We conclude by summarizing the main results and contributions.

\section{Relevant Research}

The management of human-computer conversations that incorporate emotional cues is the central area of interest for Affective Dialog Systems. This multidisciplinary field integrates work from a range of research areas, e.g., dialog processing, speech recognition, speech synthesis, computer graphics, animation, embodied conversational systems and human-computer interaction [17]. Similarly, Gebhard et al. [18] describe an approach for modeling affective states of virtual characters and explores the modeling outcomes to provide an additional resource for the generation of dialog contributions, the articulation of verbal utterances for simulated conversations among virtual characters. Traum et al. [19] present a model of virtual human agents that includes task and dialog models as well as aspects of emotional evaluation behavior, integrated to support complex team behavior such as negotiation and delegation. Mairesse et al. [20] describe a highly configurable system that generates utterances along the extroversion dimension. Using a similar approach, Campano and Sabouret [21] detail models for utterance selection based on impoliteness that consider emotions, personality and social relations.

In our approach, we focus predominantly on the text modality and on the fusion of results from naturallanguage processing and affective dialog management, applied to unrestricted open domain dialogs with users. Further, we examine the effect of the structuring of emotional interactions on perception of the system by users and its effect on the communication style of users and their expressions of affective states. The developed system is applied to online interactions and provides data that extends the scope of analysis of emotiondriven responses to online and offline events. Both the open-domain interaction settings and the characteristics of rapid online communication influenced the selection of system components presented in section 3.1. These favored robust, open domain and scalable mechanisms that are tolerant regarding the peculiarities of a chat user's approach to syntax and spelling.

Prior experimental studies on the relationship between affective states and dialog patterns observed in the interactions with Intelligent Tutoring Systems, e.g. AutoTutor, 
were presented in [22] and [23]. This line of research focuses on discovering the links between learning and emotions. The experimental results demonstrated significant correlations between the accuracy of answers and particular affective and cognitive states, e.g. "confusion" indicating inaccurate answers, "eureka" as an indicator of students learning the material and "frustration" positively correlated with the system's negative feedback and vice versa. In our work, communication content combines task-oriented dialogs specific to the interaction scenario and open domain dialogs regarding attitudes and affective responses of participants to current issues of public debate, as well as their affective states expressed during interaction with the system. A further difference to an emote-aloud method, that might affect ongoing communication processes: the presented analysis is based on automated processing and annotation of acquired conversation logs.

An early study on the effects of orientation of emotion exhibited by an embodied computer agent was presented in [24]. The experiments conducted in a Blackjack game setup investigated the psychological effects of the agent's emotions, either self-oriented or empathic, on users employing a set of pictures of actors' faces with emotional expressions and a small number of scripted responses that corresponded to game outcomes. In [25], a study on the effect of self-disclosure and empathy in text-based communication was conducted with the application of a dialog system that engaged users in closed-domain chats focused on user and system preferences regarding a set of animals. The experimental results demonstrated that increasing users agreement was the key to achieving higher closeness and user satisfaction. Similarly, self-disclosure increased when users had positive preferences for discussion topics and the system's agreement was effective for inducing agreement from users. A study focused on linguistic behavior, i.e., empathic and self-oriented emotional expression in a text-based game-setup, had similar results [26]. In particular, empathic expression significantly improved user satisfaction, raised ratings of a peer agent and increased the number of user utterances emitted in the game. Recently, evidence on the effect of turn-taking strategies on how users rate and respond to an agent was presented in [27]. These findings are first steps permitting to compare human to human communication, whether face-to-face or mediated [28], to HCI.

Exploiting these relations between affect and behavior in HCI, the developed system serves as a robust setting for studying affective human-computer interactions in real-world scenarios with a diverse set of users and for investigating the interplay between emotions and predominantly text-based communication in online affective and social dialogs. In the following, we give an overview of this method of study supported by this system.

\section{Method}

In the presented study, the Affective Dialog System is applied as a tool for studying the role of affect and social processes in HCI. The system realizes a range of interactive scenarios, e.g., by simulating different affective profiles or following fine-grained communication scenarios aimed at eliciting particular social processes from users online. Before the experiments are initiated, the participants are instructed to freely chat with the system. No additional, more specific guidelines are provided to avoid any artificial constraints on the topic selection or the communication strategy applied by experimenters. The acquired data are analyzed to improve our understanding of the role of emotions and social processes in online interaction. This approach relates to research on correlations between expressions of affect in text and physiological responses [7] as well as to studies on the relations of textual communication style and content to personality traits of users [29], [30], [31]

In this work, we present insights from three rounds of experiments:

1) Study 1 - to evaluate the system in a Wizardof-Oz (WOZ) setting in terms of its ability to generate realistic dialog, to provide an enjoyable chatting experience and to establish an emotional connection with users; to investigate to what extent affective cues can be recognized and applied in dialog management,

2) Study 2 - to investigate the role of the artificial system's affective profile on users' affective responses, emotional changes experienced during the interaction and their evaluation of the system,

3) Study 3 - to generate fine-grained communication scenarios representing key social processes, for studying their impact on users during interactions with the affective dialog system, in particular those related with changes in interaction patterns and expressions of affective states.

All of these were conducted using the system detailed below.

\subsection{Affective Dialog System}

The affective dialog system is applied to interactions with members of e-communities to probe for affective states and background knowledge related to those states [32]. The presented variants of the Affect Listener system communicate with users in a predominantly textual modality, rely on integrated affective components for detecting textual expressions of affective states, and use the acquired information to aid selection and generation of responses. Affect Listeners interact with users via a range of communication channels and interfaces that share common characteristics of online chatting.

The core tasks of the system in the context of the three experimental settings include: perception and classification of affective cues in user utterances and system 
response candidates (text-based affect detection), the incorporation of affective cues into the dialog management and the maintenance of an emotional connection with users (affective dialog management), management of task-oriented dialogs (closed-domain dialog) as well as handling conversations that are not restricted in topic (open-domain chats), and, finally, the detection of cues in the system-user interactions that enable the selection of suitable system response generation methods (balancing task oriented dialog vs. open-domain conversations). In all of the described experimental settings, the system is presented to the user in a virtual bartender scenario. In study 1 , this setting was also represented graphically by a 3D bar environment [33]. The choice of setting is intended to support interaction scenarios where direct communication with a participant can be established easily. Users can relate existing knowledge regarding the behavioral norms in such a setting in the real world to the virtual environment. Furthermore, this setting also provides the flexibility to switch between open-domain chats and closed-domain dialogs of various levels of intimacy.

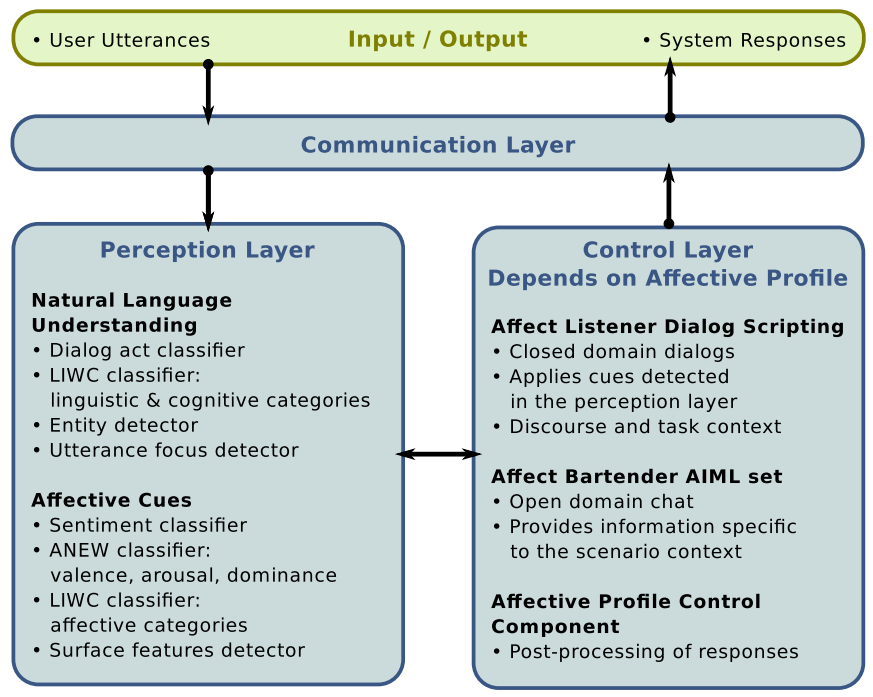

Fig. 1. Layers and components of the ADS.

In the following, we introduce the software framework and layers used in the affective dialog system, see Fig. 1. The different realizations of the system which are applied in the three rounds of experiments are developed based on the same software framework and share the same set of natural language processing tools and resources. The main difference between the system realizations used in experiments relates to their abilities to simulate distinct affective profiles or conduct finegrained social communication scenarios. All necessary modifications are implemented in the system's Control Layer. These changes affect, in particular, the ways in which different system realizations conduct taskoriented parts of the dialogs, e.g., opening or closing of the interaction. Further, they influence the system's responses depending on the applied scenario (i.e., the target communicative and affective behavior set for a given system realization) and the affective states detected in user utterances, either by suppressing potential responses to a detected affective state or by responding in a specific way, e.g. friendly or unfriendly.

Further differences between realizations of the system used in the three experimental settings are summarized in sections 4.1, 4.2 and 4.3.

\section{Perception Layer}

The Perception Layer integrates different naturallanguage processing tools, linguistic and affective resources to analyze user utterances and system response candidates:

- Dialog Act classifier: adaptation of the taxonomy used in the NPS Chat corpus [34]. For the present scenario, the original taxonomy (Accept, Bye, Clarify, Continuer, Emotion, Emphasis, Greet, No Answer, Other, Reject, Statement, Wh-Question, Yes Answer, Yes/No Question) was extended with an additional class "Order" (food or drinks). using 339 additional training instances. For this taxonomy and training set, the maximum entropy based DA classifier using a bag-of-words and bag-of-bigrams feature set achieved 10-fold cross validation accuracy of $71.2 \%$ (applied in study 1) and SVM based DA classifier 76.1\% [16] (applied in studies 2 and 3).

- LIWC (Linguistic Inquiry and Word Count) classifier [35]: provides linguistic, cognitive and affective categories for words,

- Entity detector: Gazetteers and regular expressions specific to a bar context, e.g., drinks and snacks,

- Utterance focus and interest detector [36],

- Lexicon-Based Sentiment Classifier: provides information on sentiment class, positive and negative sentiment values [37],

- ANEW classifier [38]: provides information on the valence, arousal and dominance of an utterance based on the dictionary of Affective Norms for English Words [39],

- Surface features detector: e.g., exclamation marks, emoticons.

In a nutshell, the layer is responsible for the detection of affect and other conversational cues that can be used to select suitable mechanisms and choose a system response candidate in the Control Layer. The specific set of components was selected to provide the system with the necessary cues regarding utterances required for the interaction scenarios in order to react to the participant on an affective level. This set comprises both cues on the level of content (dialog acts, LIWC categories, entities, utterance focus) as well as on the purely affective level (sentiment and ANEW values, affective LIWC categories, surface features).

\section{Control Layer}

The Control Layer manages the dialog progression by relating the observed dialog states to the intended ones, 
e.g., conducting bartender-specific tasks, querying and follow-up questions on the user's affective states, using cues acquired by the Perception Layer described above. This layer is responsible for the generation of an affectively appropriate response of the system. Whenever a response is requested, the layer selects the system's response from a number of candidates generated with: Affect Listener Dialog Scripting (ALDS) and several instruction sets for an interpreter of $\mathrm{AIML}^{2}$. Note that an empty response is a valid option in an asynchronous environment such as a chat.

Affect Listeners Dialog Scripting is an information state based dialog management component that uses a set of information cues provided by a perception layer to control dialog progression, cf. [32], [40]. The rationale for the development of ALDS is to enable interaction scenarios that provide capabilities for controlling task-oriented parts of verbal communication spanning several dialog turns, i.e., system and user utterances, and that take advantage of the system's perception capabilities, i.e., natural language analysis and affective states analysis, that extend beyond simple matching mechanisms based on keywords or textual patterns such as those provided by AIML. The ALDS scenario relies on the affective, linguistic and cognitive categories detected in a user utterance. In contrast to more complex communication tasks, e.g., close-domain dialogs aiming at acquisition of background knowledge on user's stance of expressed affective states, the application of affective cues relies on a predefined link between an initiation condition, e.g., user inputs and/or system state, and a particular system response template.

The Affect Bartender AIML set (AB-AIML) provides a robust fall-back mechanism for open domain contexts, able to generate system response candidates for a range of inputs which do not match activation cues of the provided ALDS scenarios. The adaptation of a more generic Affect Listener AIML set [32] to the experimental interaction scenarios aimed at enabling the system to generate response candidates that can: provide knowledge specific to the bartender tasks, and the virtual bar settings (all studies); convey the system's openness, interest in users' feelings, current mood, events which are of importance for them (study 1); offer a variety of responses matching different affective profiles (study 2) or supporting the realization of the fine-grained communication scenarios (study 3 ).

The Affective Profile Control Component (APCC) is used for post-processing system responses to conform to a specific affective profile ${ }^{3}$ used in the second round of experiments to convey a different affective profile of the dialog system.

2. Artificial Intelligence Markup Language (AIML)

3. Consistent affective characteristics are achieved by modifying most generated response candidates. Modifications include removing, adding or replacing discovered positive or negative expressions, words and/or emoticons. E.g., for the negative profile, the component removes phrases that contain words, classified as "positive" (e.g., glad, happy, welcome, great, sir, please).

\section{Communication Layer}

The Communication Layer provides the conversational system with an network-transparent interface to its interaction environment, e.g. events from a 3D graphics engine, Internet Relay Chat (IRC), a web-based chat, or XMPP-based chats such as Jabber, Facebook chat, or Google talk. In a distributed setup, the Communication Layer handles connections over chat-specific network protocols or a generic XML-RPC protocol. When communicating with the $3 \mathrm{D}$ event engine, the layer, in addition to the user utterances, receives and decodes arousal and valence values calculated when generating emotional facial expressions (EFE). Finally, the layer formats and dispatches system responses.

\section{INTERACTION SCENARIOS}

In the following, we describe the experimental settings and the interaction scenarios in the three studies covered.

\subsection{Study 1: Virtual Reality WOZ setting}

The focus of the first study was the evaluation of the system in comparison with a Wizard-of-Oz (WOZ) setting as a pre-requisite for the following studies. Further, we investigated the practical use of affective cues detected in user input for the generation of affective responses. As setting for the experiment, a Virtual Reality (VR) bar was created: a furnished virtual bar room and a virtual bartender and a chat interface. As the focus of the present contribution is centered around the text-based affective dialog system, we do not provide a detailed description of the technical aspects of the VR process pipelines or of the effects of emotional facial expressions displayed by the virtual bartender here. Please refer to [33] regarding those aspects.

The experimental setting consisted of the user, represented by an avatar (male or female according to the user's gender), interacting with a virtual human (male bartender). Each participant interacted four times, five minutes each, randomized order, in $2 \times 2$ conditions: The conversational partner was either a variant of the system called Affect Bartender (AB) or a Wizard of Oz (WOZ) and the generation of emotional facial expressions (EFE) was either active or not. In the $\mathrm{AB}$ condition, a simulation of thinking and typing speed was introduced to prevent an influence of differences in the response delivery time between the system and the human operator.

4. Participants believe that they communicate with a dialog system, while responses are actually provided by a human operator. In this condition, the operator adhered to general guidelines stating the objectives to be achieved during the interactions, including: providing realistic and coherent responses to the users' utterances and avoiding utterances that demonstrate an unusual sense of humor or eloquence. Several rounds of pre-test interactions helped to assure a consistency of the operator's communication patterns. 


\subsection{Study 2: Distinct Affective Profiles}

In this study, an artificial affective profile was defined as a coarse-grained simulation of affective characteristics of an individual, corresponding to dominant, observable affective traits, that can be consistently demonstrated by a system during the course of its interactions with users [15]. In this round of experiments, three distinct affective profiles were implemented for the dialog systemlabeled as positive, negative and neutral-limiting variations to baseline levels of positive and negative affectivity in personality [41]. Each affective profile aims at a consistent demonstration of character traits of the system that are described as, respectively:

- polite, cooperative, empathic, supporting, focusing on similarities with a user,

- conflicting, confronting, focusing on differences with a user,

- professional, focused on the job, not responding to expressions of affect.

The study directly addressed the main research questions about the artificial system's ability to consistently simulate affective profiles, validates the methods applied and further enables to measure their effects in interactions.

Figures 2 and 3 show excerpts of conversation logs from the study using the "positive" and "negative" affective profiles. The both logs' excerpts present also dialog parts focused on the acquisition of participants' attitude, affective states and background information related to a set of "hot topics".

The system was responsible for the management of text-based communication between the virtual bartender and a user in a browser-based web chat environment. The main objectives for the system in this interaction scenario were:

1) Realistic and coherent dialogs,

2) Conducive setting for communication, leading to the acquisition of large data sets,

3) Introducing and conducting task-oriented dialogs related to "hot topics" in order to acquire affective states of users and their stance towards the issues,

4) Maintaining a consistent affective profile (positive, negative, or neutral) throughout the whole communication with users.

The main changes compared to the previous experiments included an extensive usage of the Affective Profile Control Component (APCC) along with the application of module for Affective Profile-Dependent Postprocessing of System Responses.

\subsection{Study 3: Key Social Processes}

Affective profiles simulate human dispositional variations in baseline valence, sometimes referred to as "mood" [41] or "affective home base" [42] and are an important part of implementing different personalities. These profiles are useful for leveraging existing human
S: it is so goood to see you :) can i offer you anything to drink?

$\mathrm{U}$ : coke and lemon, as usual :)

S: here you are! enjoy! [order served]

$\mathrm{U}$ : thanks

S: you are quite welcome :) i do not see that many people in the bar at this time of a day. what are

you doing in the bar soooooo early? :)

U: late shift? :P

S: really? how do you like our bar, do you enjoy

being here? :-)

$\mathrm{U}$ : nice, cozy and good music

S: thank you :) for your kindness.

$\mathrm{U}$ : you're welcome

S: don't mention it.

U: you're very polite, even for a bartender

S: Have you heard that they want to raise

the taxes in the next year? Do you think this

decision was really necessary?

$\mathrm{U}$ : to patch up the budget, yes

$\mathrm{S}$ : What was your first reaction when you heard about this?

$\mathrm{U}$ : oh, another tax raise - that's it

S: You don't seem to be happy about this too.

Can you tell me why does this news affect you?

$\mathrm{U}$ : because we all pay taxes in some way

S: I feel very similar about it! It is a pleasure to talk with you. The next drink is on me :-)

U: my, thanks :)

S: perhaps you could tell me what did you

do today?

U: nothing, really - it's still morning :PO

S: really nothing?

$\mathrm{U}:$ ok, I ate my breakfast ;P

S: i really like talking with people like you!

Fig. 2. Dialog excerpt from the interaction with system that uses the "positive" affective profile. $\mathrm{S}=$ System; $\mathrm{U}=$ User.

communication patterns (see also section 5.2). For the next development phase of the presented dialog system, we chose two of those patterns in social human-human communication as reference points: getting acquainted and sharing of emotions. When two previously unacquainted people meet for the first time, whether online or face-to-face, they try to reduce uncertainty by "getting to know" one another. They exchange personal information, making themselves known to one another, a process called acquaintance [43]. Another important process at play when two people meet and discuss is called social sharing of emotion [44]. It consists in exchanging information about what happened to one another, and more specifically about the events which elicited emotional responses: e.g., anger towards the last public transport strike or happiness about one's latest personal success. Both processes - acquaintance and social sharing of emo- 
S: oh, no. it is you again.. what do you want

this time?

$\mathrm{U}$ : What's wrong?

S: I don't know what are you refering to. pathetic.

tell me what to you want to drink?

U: I'd like something to eat this time.

S: why do you want something to eat this time?

U: Well, i'm hungry.

S: what's your order? hurry up, you're not the only

customer in this bar....

$\mathrm{U}$ : I no, and you are not the only bartender

in this bar..

S: that's good information. you don't earn too much, do you?

$\mathrm{U}$ : Apparently, i earn enough to spend my time in this bar.

S: that is interesting:. do you want another drink?

i mean, if you have money.

you don't look as if you had..

$\mathrm{U}$ : Yes, give me 10 beers.

S: You don't sound like a person who knows that much. Anyhow.., some people say that Poland had bribed UEFA to organize UEFA Euro 2012...

Do you think we really did it?

U: Of course not. We don't have sufficent founds

to do that.

S: You are not that talkative, do you? Can you say

a bit more about you opinion on these accusations?

$\mathrm{U}$ : If there was any bribe, it has been done

by Ukrainian federation.

S: Any particular reasons for it?

$\mathrm{U}$ : They are way richer then us.

S: This was not really smart what you just said.

But I guess I shouldn't be suprised that much about it.

Fig. 3. Dialog excerpt from the interaction with system that uses the "negative" affective profile. $S=$ System; $U=$ User.

tion - are core components of everyday conversations, fostering relationship development and maintenance. They have been studied for several decades and can be elicited experimentally.

The system with the neutral affective profile applied in the study 2 was used both as a reference baseline system as well as a starting point for the development of system variants suitable for these fine-grained communication scenarios. The conceptualization and experimental evaluation of these realizations of the dialog system enabled to further address the main research questions set for all the presented studies. These in particular related to the artificial system's ability to convincingly conduct fine-grained social communication scenarios, validate the methods applied and measure the effects of the introduced scenarios in interactions with participants.

\subsubsection{Communication scenario: getting acquainted}

In this scenario, the system aims at acquiring personal information about the user and demonstrating reciprocity in sharing personal information. The system starts with low-intimacy topics and questions, appropriate for the student population that formed the participant pool for this study, e.g., "What is your major?", "What kind of music do you like?". During the course of the interaction, the system progressively introduces more intimate topics, e.g., "Is anything making you stressed out these days?" (mild intimacy), "What is your most frightening memory?" (high intimacy). Reciprocity in sharing information is achieved by revealing info about itself, e.g., common interests with user, as well as other personal likes and dislikes. These are frequently introduced at the beginning of a system utterance, followed with a question related to the same topic, e.g., "I really like math. What is your favorite class?". This experimental setup resembles a real-world communication scenario, typical for initiating and developing contact with an unknown person by exchanging information of increasing intimacy. The list of questions of various levels of intimacy was adapted from [45].

Implementation of the scenario was achieved by modification of the AIML sets used in the Affect Bartender system variant and the introduction of goal-oriented dialog elements, implemented in the form of ALDS commands. Specifically, the base AIML set was modified to include the set of questions commonly used when establishing contact. Further, we introduced triggers that related three classes of questions with increasing intimacy level (low, mild, high) to dialog progression as gauged by the number of utterances exchanged with the user. The ALDS-based communication scenarios were focused on two topics: conversation about the country of origin of participants and their feelings towards it (initial part of the interaction), and implicit feedback related to the enjoyment of the so-far-conducted conversation with the bartender (closing part of the interaction). Fig. 4 shows an excerpt of a conversation log in the "getting acquainted" communication scenario.

\subsubsection{Communication scenario: social sharing of emo- tion}

In this scenario, after conducting the initial part of dialog related to ordering drinks, the Affect Bartender shares an emotionally-rich story from "personal" experience which is presented in a negative context (see Fig. 5). Once this part is completed, the system asks the user to recall an intense emotional experience and tries to gather more details about this experience e.g., time, place, duration, feelings, or previous sharing.

Similarly to the previous communication scenario, the implementation was realized by a modification of the AIML sets and the introduction of ALDS-based communication scenarios. Specifically, the ALDS-based scenarios were used to introduce the emotionally rich episode 


S: Where do you come from?
U: i am from bulgaria
S: I like bulgaria. When you are away,
do you miss it?
U: i miss my friends and family, but, yeah,
the place as well
S: Why is it like that, why do you feel this way?
U: because they are every special for me,
and moreover i cannot find my place here yet
S: I understand. As for me I really like
being here. People are really nice :)
U: they are, indeed. but it takes time to get to
get to know them and get used to everything

Fig. 4. Dialog excerpt from the "getting acquainted" communication scenario. $\mathrm{S}=$ System; $\mathrm{U}=$ User.

S: This morning I was sooo angry at a customer.
U: what did he do?
S: He tried to leave without paying.
U: oh, that is really bad
S: Yes. What was the last time you were angry at
someone? Can you tell me what happened then?
U: the last time i was angry when i attended
the USG parlamentarien meeting
S: can you tell me more about it?
U: these people are so selfish and opionated.
they only accepted their own point of view

Fig. 5. Dialog excerpt from the "social sharing of emotion" communication scenario. $\mathrm{S}=$ System; $\mathrm{U}=$ User.

that is recalled by the bartender and extended in the next part with a set of questions aimed at encouraging the user to share a similar emotionally intense experience. Towards the end of communication in the "social sharing of emotion" scenario, another ALDS-based dialog is used to recall the previously-mentioned experience of the user. The base AIML set was modified to more frequently include questions related to users' emotions, previous sharing of the emotionally-rich experience recalled, and assessing the influence of such an experience on the current emotional state of the user.

\section{EXPERIMENTAL RESULtS}

This section summarizes the evaluation results of the first two studies and adds an analysis of the results from the third one, i.e., the effect of fine-grained communication scenarios on the participants communication style, on the textual expression of affective states and on self-reported experience of emotional changes during interaction. We also describe general findings obtained through analyzing the aggregated data-sets with natural- language and affect processing tools ${ }^{5}$, differences in system's communication style and expressions of affective states in the three studies.

\subsection{Study 1}

Interactions in all four experimental settings $(2 \times \mathrm{WOZ}$, 2 x System) were completed by 35 participants (13 female, 22 male), aged between 20 and 50, resulting in 140 interaction logs. English, the language in which the experiments were conducted, was not the native language for all participants, but all had at least good communicative skills.

After each of the experimental interactions, lasting 5 minutes, participants were asked the following questions for assessing the conversational system $(\mathrm{VH}=$ Virtual Human, the label used for the graphical bartender avatar):

1) Did you find the dialog with the VH to be realistic?

2) How did you enjoy chatting with the VH?

3) Did you find a kind of emotional connection between you and the VH?

The participants provided their ratings on a six-point Likert scale, i.e., from $1=$ not at all to $6=$ very much. Figure 6 presents the aggregated results obtained for the experimental settings with the Affect Bartender and for those with a Wizard-of-Oz ${ }^{6}$. In all 3 tasks, the results achieved by the conversational system match those obtained for the WOZ. In particular, the correlation coefficients for the aggregated $\mathrm{AB}$ and $\mathrm{WOZ}$ ratings varied between .95 (chatting enjoyment), .96 (emotional connection) and .97 (dialog realism). All these correlations differ from 0 at a significance level of .001. A repeated measures analysis of variance showed no main effect of the setting ( $\mathrm{AB}$ vs. WOZ) on the three dependent measures (all Fs $(1,34)<.50$, ps $>.49$ ). Pairwise comparisons with Bonferroni correction confirm the absence of significant differences between the two settings on the perception of dialog realism, chatting enjoyment, and subjective feeling of emotional connection with the system.

\subsubsection{Comparisons Between System and WOZ Data}

Comparisons between system and WOZ utterances demonstrate success in conveying interest in feelings and concerns of users, potentially connecting with the user. Specifically, examined with the lexicon-based sentiment classifier, system utterances were both more positive,

5. Linguistic Inquiry and Word Count dictionary, ANEW dictionarybased classifier, Lexicon-Based Sentiment Classifier, and Support Vector Machine Based Dialog Act classifier. Further, we analyzed timing information and surface features of communication style such as wordiness and usage of emoticons. Correct detection of all expressions of affect, linguistic and discourse-related cues cannot be guaranteed, however, this set of tools and resources has been successfully applied in numerous psychological experiments and extensively evaluated and validated [35], [38], [39], [40], [46], [47], [48], [49] supporting their application for the automatic analysis of text in different domains.

6. In all figures, data are normalized by the number of utterances emitted by a user in a given interaction. Asterisks indicate significant differences at $\mathrm{p}<.05$. Error bars indicate \pm 1 standard error. 


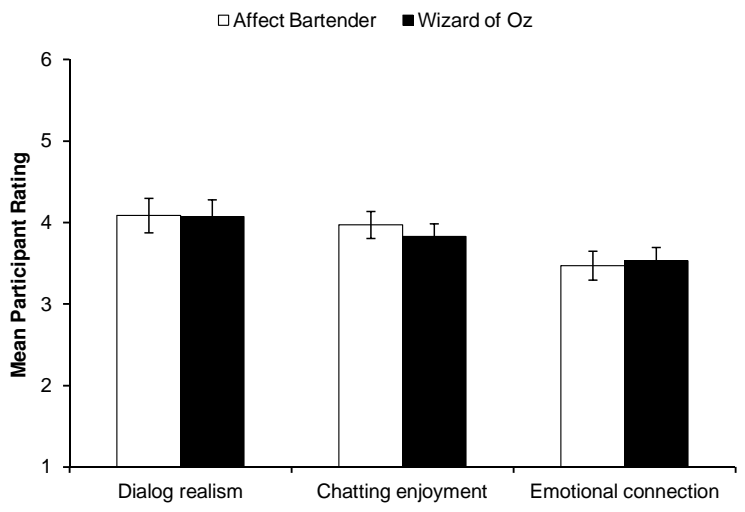

Fig. 6. Study 1: System vs. WOZ - Evaluation Results

and more negative compared to WOZ utterances (repeated measures analysis of variance, $\operatorname{Fs}(1,34)>36.61$, ps < .001). Simply put, system utterances were more emotionally loaded than WOZ utterances, both in the positive and in the negative orientation. Additionally, when examining utterances with LIWC personal concerns categories, we find that the system generated significantly more words related to work, home, money, religion, and death, compared to the $\mathrm{WOZ}(\mathrm{Fs}(1,34)>$ 11.59 , ps < .01). In short, the system was able to talk more about emotions as well as potential user concerns, in an attempt to relate to user's feelings and interests.

\subsection{Study 2}

For conducting the second study, a browser-based communication interface, resembling a typical web chatroom environment was developed: a user input field at the bottom of the screen and a log of communication in the center. Participants interacted with all three affective profiles (positive, neutral and negative) in turn, once with each. To avoid ordering effects in the evaluation of the different system realizations, the actual sequence was randomly and evenly assigned and the evaluation statements were also displayed to users before the start of the first interaction to familiarize themselves with rating. These statements were:

1) I enjoyed chatting with the conversational partner during the just completed interaction.

2) I found a kind of "emotional connection" between myself and the conversational partner.

3) I found the dialog with the conversational partner to be realistic.

4) I found the dialog to be coherent. In other words, the sequence of responses of the conversational partner made sense.

5) I noticed a positive emotional change in myself during the interaction.

6) I noticed a negative emotional change in myself during the interaction.

7) I would like to chat again with this particular conversational partner in the future.
During the experiments, after each experimental condition corresponding to a single affective profile, participants were asked to express their agreement or disagreement with the statements on a five-point Likert scale, i.e., from $1=$ strongly disagree to $5=$ strongly agree. Participants interacted with the dialog system in an unsupervised manner and were aware that they were talking with an artificial system. Interactions were always initiated by the system, i.e. the system provided the first utterance, and stopped after 7 minutes with a suitable closing response, followed by the display of the questionnaire. To further increase the number of system-user message exchanges compared to previous experiments, no artificial delays, e.g., a simulation of thinking or typing, were used in this experiment.

Interactions were completed by 91 participants (33 female, 58 male), aged between 18 and 52, in all three experimental settings resulting in 273 interaction logs. English, the language in which the experiments were conducted, was not the native language for all participants, but all participants had at least average communication skills in this language.

\subsubsection{Effects of Affective Profile on System's Evaluation and Emotional Changes}

The affective profile had a series of significant effects on the evaluation of the system and on users' emotional changes. Detailed results of the analyses performed are presented in [15] and [16]. Concerning evaluation, the affective profile had significant effects on all dependent measures (see Figure 7). The largest effect sizes were found on statements 5 and 6 (positive and negative emotional change, respectively). As expected, affective profiles successfully induced corresponding emotional changes in users, affecting perception of dialog realism and coherence only to a smaller extent.

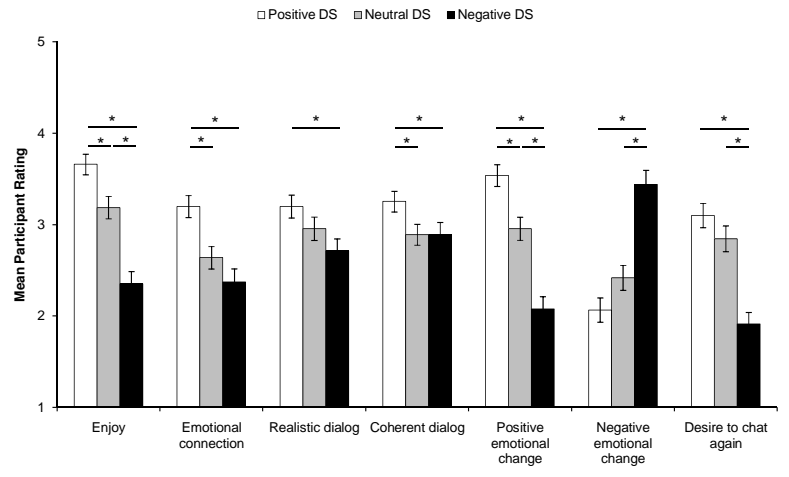

Fig. 7. Participant's mean ratings on all dependent variables, of their interactions with the dialog system (DS) with three different affective profiles (positive, neutral, negative).

\subsubsection{Effects of Affective Profile on Users' Interaction Style}

Users were equally fast in replying to different affective profiles. Specifically, when analyzing the whole inter- 
actions, there were no differences in the participants' average response time to a number of letters generated. They also used an equal amount of words and utterances in their conversations for all profiles. There were, however, significant differences in word categories used and other linguistic aspects of the text input. Among others, compared with the positive profile, the negative profile elicited, as expected, less assent (e.g., ok, yes, agree) from users, fewer positive emotion words, more anger-related words, and utterances assessed as significantly less positive by the sentiment and ANEW classifiers (see Figure 8). The positive profile, on the other hand, elicited accordingly more positive emoticons, more positive emotion words (e.g., love, nice, sweet), more user statements, and less closed questions to the system. The two latter findings might indicate more information disclosure and less questioning from users towards the positive profile, compared to the negative profile.

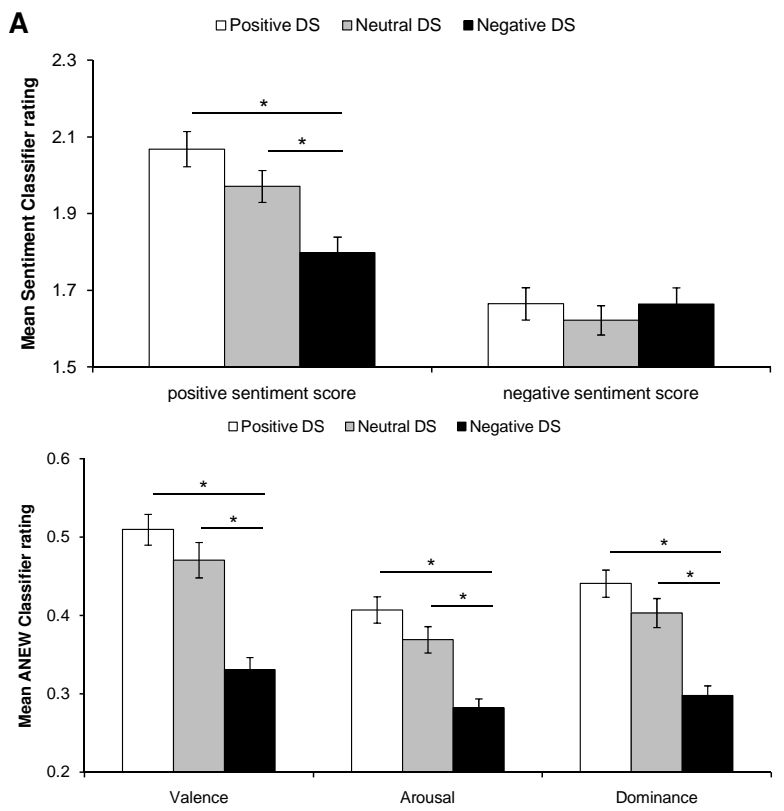

Fig. 8. Valence, arousal, and dominance ratings in user exchanges with the dialog system (DS). Panel A shows the mean positive and negative Sentiment Classifier score per condition. Panel B shows the mean valence, arousal, and dominance scores based on the ANEW lexicon.

\subsection{Study 3}

The experimental setup and procedure were similar to those of Study 2. In particular, the browser-based interface resembling a typical web chat-room was used. Users were also informed that they would interact with a dialog system, and each interaction was restricted to 7 minutes. During the study, after each experimental condition corresponding to a particular scenario, participants were asked to evaluate the system on the seven items used in Study 2.
Interactions were completed by 75 participants (38 female, 37 male), aged between 18 and 32, in all three experimental settings resulting in 225 interaction logs. Participants were native English speakers $(\mathrm{n}=18)$ or had proven English proficiency ( $\mathrm{n}=57$, passed TOEFL(C). After initial analysis of the data-set, two outliers were excluded due to scores beyond three standard deviations from the mean on different scales. The final sample therefore consists of 73 participants.

\subsubsection{Effect of Communication Scenario on System's Evaluation}

A multivariate repeated measures analysis of variance showed the expected absence of effect of communication scenario on users' evaluations of the system (Wilks' $\lambda=.90, \mathrm{~F}(14,276)=1.07, \mathrm{p}=.39)$. In other terms, participants judged the three communication scenarios (neutral, getting acquainted, and social sharing of emotion) as equally enjoyable, coherent, realistic, emotionally connecting, etc. (univariate tests: all $\mathrm{Fs}(2,144)<2.47$, ps $>$.09).

\subsubsection{Effects of Communication Scenario on Users' In- teraction Style}

Words and Timing. Participants conversed significantly more in the "social sharing of emotion" scenario, despite the time limitation. There was a main multivariate effect of communication scenario on indicators of conversation length (Wilks' $\lambda=.84, \mathrm{~F}(6,284)=4.48, \mathrm{p}<.001$ ). Univariate analysis showed that each length indicator, i.e., character, word, and utterance count, was affected by the communication scenario $(\operatorname{Fs}(2,144)>3.03$, ps $\leq$ .05). Post-hoc comparisons, shown in Table 1, revealed that participants wrote significantly more when sharing an emotional episode.

Confirming these results, we found a main effect of communication scenario on response time $(\mathrm{F}(2,144)=$ $8.29, \mathrm{p}<.001)$. Specifically, participants wrote significantly faster in the social sharing of emotion scenario, compared to the two other conditions (see Table 1).

\begin{tabular}{|c|c|c|c|c|c|c|}
\hline \multirow[b]{3}{*}{ Variable } & \multicolumn{6}{|c|}{ Communication Scenario } \\
\hline & \multicolumn{2}{|c|}{ BASE } & \multicolumn{2}{|c|}{$\mathrm{ACQ}$} & \multicolumn{2}{|c|}{ SHARE } \\
\hline & M & $S D$ & M & $S D$ & M & $S D$ \\
\hline Character Count & $810.92 \mathrm{a}$ & 222.53 & $788.10_{\mathrm{ab}}$ & 193.80 & $828.10 \mathrm{ac}$ & 187.64 \\
\hline Word Count & $164.03 \mathrm{a}$ & 42.65 & $160.78 \mathrm{ab}$ & 38.37 & $171.60 \mathrm{ac}$ & 36.02 \\
\hline Utterance Count & $26.82 \mathrm{ab}$ & 10.05 & 27.33 a & 9.28 & $29.32 \mathrm{ac}$ & 11.69 \\
\hline Response Time & $2.57 \mathrm{a}$ & .76 & $2.62 \mathrm{a}$ & .65 & $2.39 \mathrm{~b}$ & .56 \\
\hline
\end{tabular}

Notes: $N=73$. For each row, different subscripts indicate significant differences at $p<.05$ (pairwise comparisons with Bonferroni correction). Response time is standardized with the number of characters scenario: SHARE = social sharing of emotion communication scenario.

Dialog Act classes. Participants wrote significantly less statements in the social sharing scenario, and significantly more orders in the neutral scenario $(\mathrm{Fs}(2$, $144)>5.63$, ps $<.01)$. 


\subsubsection{Effect of Communication Scenario on User's Ex- pression of Affective States}

Sentiment Classifier, and ANEW lexicon. There was a main effect of communication scenario on both positive sentiment score $(F(2,144)=5.59, \mathrm{p}<.001)$ and ANEW ratings (Wilks' $\lambda=.60, \mathrm{~F}(6,284)=13.77, \mathrm{p}<.001)$. As depicted in Figure 9, participants wrote significantly less positive, less arousing, and less dominant utterances in the social sharing of emotion scenario; note the negative context of the sharing introduced by the system's "personal experience". The largest effect size is found for ANEW valence ratings $\left(\eta_{p}^{2}=.22\right)$ first indication of the successful creation of a social sharing of emotion situation, where participants shared a negative experience, which did not happen in the two other conditions.

\section{A}
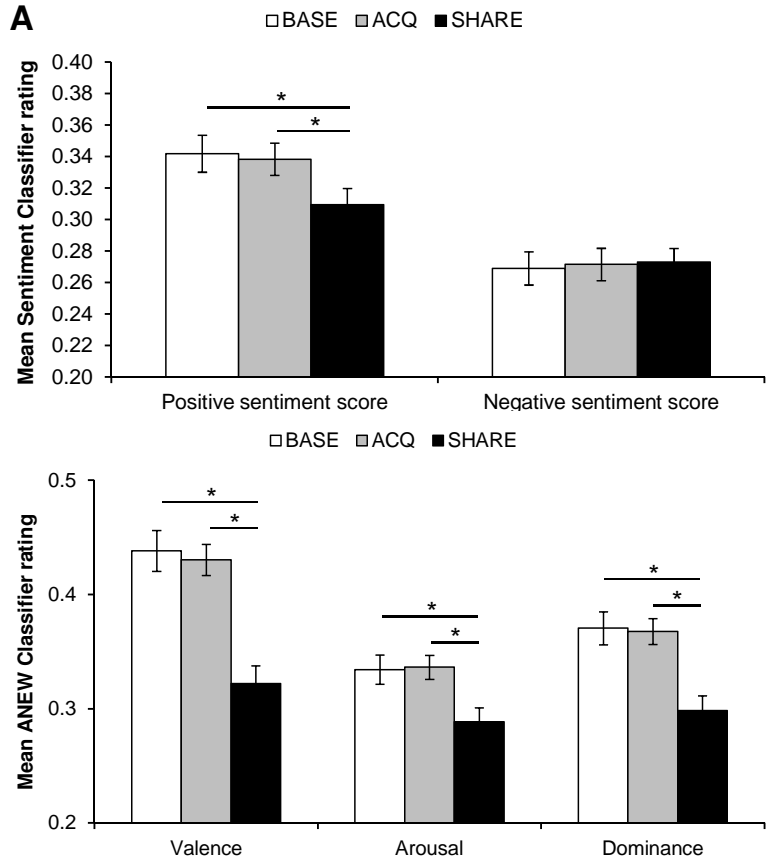

Fig. 9. Study 3: Effect on Expressions of Affective States and Sentiment

LIWC variables. The pattern of results obtained with LIWC variables also indicates successful creation of a social sharing of emotion situation in that respective communication scenario. Specifically, participants in that scenario used significantly more function words and pronouns, referring more frequently in their utterances to social relations and people $(\mathrm{Fs}(2,144)>7.63$, ps $<.01)$. The biggest effect sizes are found with the use of 3 rd person singular words (e.g., she, her, him; $\eta_{p}^{2}=.30$ ), to relate to another person, and use of past tense conjugation $\left(\eta_{p}^{2}=.41\right)$ : participants talked about something that happened in the past, in relation to another person. They also talked about something significantly more negative, containing significantly more anger-related words $(\mathrm{Fs}(2$, $144)>16.45$, ps $<.001$ ). This suggests that this communication scenario was able to elicit the social sharing of an episode of anger from the user. To confirm the obtained results, manual content analysis was performed as well.

\subsubsection{Manual Content Analysis}

Two independent judges rated the extent of disclosure of personal and emotional information in all conversation logs. Self-disclosure was defined as "the voluntary and verbal communication of personal information" [50], and measured on two dimensions: frequency and depth. Frequency referred to the number of utterances containing disclosure of personal information, normalized with the number of utterances emitted by the user. Depth referred to the intimacy of the information divulged and was measured on a ten-point Likert scale (from $1=$ no intimate information divulged to $10=$ highly intimate information divulged).

Social sharing of emotion was defined as "the voluntary and verbal communication of one specific past emotional episode" [44]. Again, two dimensions labeled frequency and depth were measured. Sharing frequency consisted in the presence or absence of social sharing in a conversation. Sharing depth consisted in a count of the utterances containing instances of social sharing within a conversation. Mean inter-rater agreement for all four measures was $.87(\operatorname{ICC}(2, \mathrm{k}))$.

Analyses of the manual content ratings confirm the successful elicitation of both processes within their respective communication scenarios. Specifically, we found a main effect of communication scenarios on all manual measurements $(\mathrm{Fs}(2,144)>49.30$, ps $<.001)$. As shown in Table 2, users disclosed personal information significantly more frequently in the acquaintance scenario. Disclosure depth, however, was equally high in both scenarios, acquaintance and social sharing. Concerning measures of social sharing of emotion, both frequency and depth reveal the successful and unique generation of this process in its respective communication scenario.

TABLE 2: Means and standard deviations of judges' ratings of self-disclosure and social sharing of emotion, per conversation with each communication scenario

\begin{tabular}{|c|c|c|c|c|c|c|}
\hline \multirow[b]{3}{*}{ Variable } & \multicolumn{6}{|c|}{ Communication Scenario } \\
\hline & \multicolumn{2}{|c|}{ BASE } & \multicolumn{2}{|c|}{$A C Q$} & \multicolumn{2}{|c|}{ SHARE } \\
\hline & M & $S D$ & M & $S D$ & M & $S D$ \\
\hline SDI Frequency & $.17 \mathrm{a}$ & .08 & $.30 \mathrm{~b}$ & .13 & .17a & .10 \\
\hline SDI Depth & $1.52 \mathrm{a}$ & .36 & $1.88 \mathrm{~b}$ & .43 & $1.84 \mathrm{~b}$ & .46 \\
\hline SSE Frequency & $.05 \mathrm{a}$ & .20 & $.27 \mathrm{~b}$ & .41 & $.74 \mathrm{c}$ & .42 \\
\hline SSE Depth & $.18 \mathrm{a}$ & 1.03 & $.59 a$ & 1.23 & $2.45 \mathrm{~b}$ & 1.94 \\
\hline
\end{tabular}

Notes: $N=73$. For each row, different subscripts indicate significant differences at $p<.05$ (pairwise comparisons with Bonferroni correction). BASE = neutral communication scenario; $\mathrm{ACQ}=$ gettinc Self-Disclosure; SSE = Social Sharing of Emotion.

\section{General Findings ANd Discussion}

The main research questions addressed in this work relate to the ability of a text-based dialog system to simulate affective profiles, realizing a range of fine-grained communication scenarios and to testing the effects of this simulation in interactions. Both the evaluation results and the analysis of the participants' communication style and expressions of affective states obtained from all three rounds of experiments support the thesis that the affective dialog system, although restricted to the text 
modality, can convincingly simulate an affective profile and realize different social communication scenarios in short interactions. The conducted experiments also provide strong evidence on the effect of affective and social processes simulated in the affective dialog system on users' communication style and textual expressions of affective states, even when the communication is limited only to the textual modality and participants are aware they interact with an artificial system.

In addition, the analysis of the results from three studies reveals interesting differences caused by the chosen interaction scenarios. Specifically, statistically significant differences in wordiness and users' response time observed in Study 3, i.e. Social Sharing vs. Acquaintance, Social Sharing vs. Base System, were not recorded in Study 2. The scenario for Social Sharing of a personal negative emotional experience, introduced in Study 3 had an effect on affective states expressed by participants similar to the one observed in Study 2 with a dialog system equipped with the negative affective profile. Both scenarios led to lower valence, arousal and dominance detected in users' utterances (ANEW). A similar effect was also observed in the expressions of positive sentiment and usage of positive statements (based on the Sentiment Classifier). On the other hand, the application of affective profiles in Study 2, also elicited changes in the usage of neutral utterances and emoticons, which were not observed in Study 3. Compared to Study 2, the finegrained social communication scenarios used in Study 3 did not influence the perception of core capacities of the system, i.e., dialog realism and dialog coherence. There were no differences in the rankings of enjoyment or desire to chat again with the system. Furthermore, in Study 3, no statistically significant differences were discovered for the participants' subjective feeling of positive or negative emotional change. In contrast, the variety of affective profiles simulated by the system in Study 2 strongly influenced all off the above presented measurements. Additionally, the variety of interaction environments (VR and online chat), experimental setups (laboratory and remote participation) and experiment participants' profiles (i.e., sampling differences between studies), while providing high environmental validity for the study, restricts the ability to statistically compare study results beyond the obtained effect sizes. Results can not be disentangled to find differences due to external factors vs. differences due to variations in dialog system design.

The influence of emotions on communication processes extends to the virtual setups. People treat interactions with certain media (including types of virtual agents) similarly to real humans [51]. For example [52] demonstrated that affective mood influences the disclosure of personal information both in real and virtual setups, providing evidence for the transfer of experimental results between HCI setups and human-human interactions. Such disclosure is an essential part of human relationship formation [43]. Other aspects relevant to the practical exploration of results presented in this article relate to people's increased willingness to cooperate more with agents that are more human-like (extended version of mechanisms similar to kin selection [53]), and the influence of emotional expressions of agents on engagement [54], [55]. Further, participants pay attention to social cues [56] and the social function of emotions could also be observed in human-agent interactions [57], [58].

\section{Conclusions}

In this paper, we presented an integrated view on a series of experiments conducted with the Affective Dialog System. The system was applied as a tool for studying communication processes and the role and impact of affect and social processes on users in online interactions that are predominantly text-based. Regarding system design and implementation, we focused on challenges of structuring emotional interactions in open-domain dialogs limited to the textual modality, and on investigating the impact of such interactions on users. The first study, using a WOZ setting, validated the system's ability, on par with a human operator regarding realistic and enjoyable dialog as well as for establishing an emotional connection with users in a short interaction. The second study showed a significant influence of the system's affective profile on users' perception of a conversational partner, on the changes of emotional states reported by the users, and on the textual expressions of affective states. The third study demonstrated a successful application of the dialog system for eliciting social sharing of emotion and for realizing a communication scenario of 'getting acquainted'. The experimental results show the impact of the realized communication scenario on the communication style of users and on expressions of affective states, without affecting the overall perception of the conversational system.

The presented studies relate to a number of research questions that are of concern for the development of interactive affective systems: How do emotions manifest in a textual form in specific interaction setups? How can the affective profile of a conversational partner influence emotional states? How do we communicate these changes online? Is there a difference between human and artificial interaction partners regarding affective impact? The results presented in this article demonstrate that affective dialog systems are well suited as tools for studying those questions by simulating communication behavior as plausible "online characters" and recording the impact on users. The obtained results are in line with findings from psychological studies that examined the role of affect and social processes in human-human interaction, both in the laboratory and in natural, potentially ICT-mediated communication settings.

Our future research includes extended investigation of the effect of emotions and social processes in user-system interactions, in particular in environments with multiple 
simultaneous users. Studies will examine the relations between communication style, textual expressions of affective, social and cognitive processes and physiological responses of users in online dialogs. Another promising area of application of affective dialog systems is interactive study and support of online communities [59], e.g., by online analysis of the affective state of a group using simulations of group dynamics.

\section{ACKNOWLEDGMENTS}

The Austrian Research Institute for AI (OFAI) is supported by the Austrian Federal Ministry for Transport, Innovation, and Technology. Further support was received from the European Community's 7th Framework Program under grant no 231323 (CYBEREMOTIONS).

\section{References}

[1] Y. Marzouki, I. Skandrani-Marzouki, M. Bejaoui, H. Hammoudi, and T. Bellaj, "The contribution of facebook to the 2011 tunesian revolution: A cyberpsychological insight," Cyberpsychology, Behavior and Social Networking, vol. 15, no. 5, 2012.

[2] M. Mitrovic and B. Tadic, "Patterns of emotional blogging and emergence of communities: Agent-based model on bipartite networks," arXiv, 2011. [Online]. Available: http://arxiv.org/ pdf $/ 1110.5057$

[3] A. Chmiel, J. Sienkiewicz, M. Thelwall, G. Paltoglou, K. Buckley, A. Kappas, and J. Holyst, "Collective emotions online and their influence on community life," PLoS ONE, vol. 6, no. 7, p. e22207, 2011.

[4] M. Thelwall, K. Buckley, and G. Paltoglou, "Sentiment in twitter events," J. of the American Society for Information Science and Technology, vol. 1, no. 63, pp. 163-173, 2012.

[5] R. Hillmann and M. Trier, "Sentiment polarization and balance among users in online social networks," in Proc. 18th Americas Conference on Information Systems AMCIS 2012, Seattle WA, 2012.

[6] A. Garas, D. Garcia, M. Skowron, and F. Schweitzer, "Emotional persistence in online chatting communities," Scientific Reports, vol. 2, no. 402, 2012.

[7] A. Kappas, D. Kuester, M. Theunis, and E. Tsankova, "Cyberemotions: Subjective and physiological responses to reading online discussion forums," in 50th Annual Meeting of the Society for Psychophysiological Research, Portland OR, 2010.

[8] D. Kuester and A. Kappas, "Measuring emotions in individuals and internet communities," in Internet and Emotions, T. Benski and E. Fisher, Eds. Research Network of the European Sociological Association, 2013.

[9] S. Turkle, The second self: computers and the human spirit. New York, NY, USA: Simon \& Schuster, Inc., 1984.

[10] - Alone together: Why we expect more from technology and less from each other. New York, NY, USA: Basic Books, 2011.

[11] R. Calvo and S. D'Mello, "Affect detection: An interdisciplinary review of models, methods, and their applications," IEEE Transactions of Affective Computing, vol. 1, pp. 18-37, 2010.

[12] S. Marsella, J. Gratch, and P. Petta, "Computational Models of Emotion," in A Blueprint for Affective Computing - A sourcebook and manual. Oxford Univ. Press, 2010, pp. 21-46.

[13] P. Petta, C. Pelachaud, and R. Cowie, Eds., Emotion-Oriented Systems: The Humaine Handbook, ser. Cognitive Technologies Series. Springer, Berlin/Heidelberg/New York, 2011.

[14] M. Skowron, H. Pirker, S. Rank, G. Paltoglou, J. Ahn, and S. Gobron, "No peanuts! affective cues for the virtual bartender," in Proc. of the Florida Artificial Intelligence Research Society Conf. FLAIRS, Palm Beach FL. AAAI Press, 2011.

[15] M. Skowron, S. Rank, M. Theunis, and J. Sienkiewicz, "The good, the bad and the neutral: affective profile in dialog system-user communication," in Proc. 4th bi-annual Affective Computing and Intelligent Interaction Conference, Memphis TN, ser. LNCS. Springer, 2011, pp. 337-346.
[16] M. Skowron, M. Theunis, S. Rank, and A. Borowiec, "Effect of affective profile on communication patterns and affective expressions in interactions with a dialog system," in Proc. 4th bi-annual Affective Computing and Intelligent Interaction Conference, Memphis TN, ser. LNCS. Springer, 2011, pp. 347-356.

[17] E. Andre, L. Dybkjaer, W. Minker, and P. Heisterkamp, Eds. Affective Dialogue Systems, Tutorial and Research Workshop (ADS 2004), ser. LNAI 3068. Springer Berlin-Heidelberg, 2004.

[18] P. Gebhard, M. Klesen, and T. Rist, "Coloring multi-character conversations through the expression of emotions," in Affective Dialogue Systems Workshop, Irsee Germany, 2004, pp. 128-141.

[19] D. R. Traum, J. Rickel, J. Gratch, and S. Marsella, "Negotiation over tasks in hybrid human-agent teams for simulation-based training," in AAMAS, Melbourne Australia, 2003, pp. 441-448.

[20] F. Mairesse, M. Walker, M. Mehl, and R. Moore, "Using linguistic cues for the automatic recognition of personality in conversation and text," J. of AI Research, vol. 30, pp. 457-500, 2007.

[21] S. Campano and N. Sabouret, "A socio-emotional model of impoliteness for non-player characters," in AAMAS, Budapest Hungary, 2009, pp. 1123-1124.

[22] S. D'Mello, S. Craig, B. Gholson, S. Franklin, R. Picard, and A. Graesser, "Integrating affect sensors in an intelligent tutoring system," in Affective Interactions: The Computer in the Affective Loop Workshop at 2005 Int.Conf.on Intelligent User Interfaces. AMC Press, 2005, pp. 7-13.

[23] A. Graesser, S. D'Mello, S. Craig, A. Whiterspoon, J. Sullins, B. McDaniel, and B. Gholson, "The relationship between affective states and dialog patterns during interactions with autotutor," $J$. of Interactive Learning Research, vol. 2, no. 19, pp. 293-312, 2008.

[24] S. Brave, C. Nass, and K. Hutchinson, "Computers that care: investigating the effects of orientation of emotion exhibited by an embodied computer agent," Int. J. Hum.-Comput. Stud., vol. 62, pp. 161-178, 2005.

[25] R. Higashinaka, K. Dohsaka, and H. Isozaki, "Effects of selfdisclosure and empathy in human-computer dialogue," in Proc. of IEEE Workshop on Spoken Language Technology, Goa India, 2008, pp. $109-112$

[26] K. Dohsaka, R. Asai, R. Higashinaka, Y. Minami, and E. Maeda, "Effects of conversational agents on human communication in thought-evoking multi-party dialogues," in SIGDIAL Meeting, London UK, 2009, pp. 217-224.

[27] M. ter Maat, K. P. Truong, and D. Heylen, "How agents' turntaking strategies influence impressions and response behaviors," Presence, vol. 20, no. 5, pp. 412-430, 2011.

[28] A. Kappas and N. Kramer, Face-to-Face Communication Over the Internet: Emotions in a Web of Culture, Language and Technology. Cambridge University Press, 2011.

[29] J. Pennebaker and L. King, "Linguistic styles: Language use as an individual difference," J. of Personality and Social Psychology, vol. 77, no. 6, pp. 1296-1312, 1999.

[30] L. Fast and D. Funder, "Personality as manifest in word use: Correlations with self-report, acquaintance report, and behavior," J. of Personality and Social Psychology, vol. 94, no. 2, pp. 334-346, 2008.

[31] T. Yarkoni, "Personality in 100,000 words: A large-scale analysis of personality and word use among bloggers," J. of Research in Personality, vol. 44, no. 3, pp. 363-373, 2010.

[32] M. Skowron, "Affect listeners. acquisition of affective states by means of conversational systems," in Development of Multimodal Interfaces - Active Listening and Synchrony, ser. LNCS. Springer, 2010, pp. 169-181.

[33] S. Gobron, J. Ahn, S. Quentin, D. Thalmann, M. Skowron, S. Rank, G. Paltoglou, and M. Thelwall, "An interdisciplinary vrarchitecture for 3d chatting with non-verbal communication," in Proc. of Joint Virtual Reality Conf. of EuroVR, Nottingham UK, 2011.

[34] E. Forsyth and C. Martell, "Lexical and discourse analysis of online chat dialog," in Proc. of the First IEEE Int. Conf. on Semantic Computing, 2007, pp. 19-26.

[35] J. W. Pennebaker, M. E. Francis, and R. K. Booth, Linguistic Inquiry and Word Count: LIWC 2001. Erlbaum Publishers, 2001.

[36] M. Skowron, J. Irran, and B. Krenn, "Computational framework for and the realization of cognitive agents providing intelligent assistance capabilities," in 6th Int. Cognitive Robotics Workshop, at ECAI, Patras Greece, 2008, pp. 88-96.

[37] G. Paltoglou, S. Gobron, M. Skowron, M. Thelwall, and D. Thalmann, "Sentiment analysis of informal textual communication in cyberspace," in Proc. Engage 2010, Zermatt CH, 2010, pp. 13-25. 
[38] G. Paltoglou, M. Theunis, A. Kappas, and M. Thelwall, "Predicting emotional responses to long informal text," IEEE Transactions on Affective Computing, vol. Advance online publication, pp. 1-11, 2012.

[39] M. Bradley and P. Lang, "Affective norms for english words (anew): Stimuli, instruction manual and affective ratings," Center for Research in Psychophysiology, Univ. of Florida, Tech. Rep. C1, 1999.

[40] M. Skowron and G. Paltoglou, "Affect bartender - affective cues and their application in a conversational agent," in IEEE Symposium Series on Computational Intelligence 2011, Workshop on Affective Computational Intelligence. IEEE, 2011.

[41] D. Watson and A. Tellegen, "Toward a consensual structure of mood," Psychological Bulletin, vol. 98, no. 2, pp. 219-235, 1985.

[42] P. Kuppens, Z. Oravecz, and F. Tuerlinckx, "Feelings change: Accounting for individual differences in the temporal dynamics of affect," J. of Personality and Social Psychology, vol. 99, no. 6, pp. $1042-1060,2010$

[43] I. Altman and D. Taylor, Social penetration: The development of interpersonal relationships. Holt, Rinehart \& Winston, 1973.

[44] B. Rime, "Emotion elicits the social sharing of emotion: Theory and empirical review," Emotion Review, vol. 1, no. 1, pp. 60-85, 2009.

[45] C. Sedikides, W. Campbell, G. Reeder, and A. Elliot, "The relationship closeness induction task," Representative Research in Social Psychology, vol. 23, pp. 1-4, 1999.

[46] J. W. Pennebaker, M. Mehl, and K. Niederhoffer, "Psychological aspects of natural language use: Our words, our self," Annual Review of Psychology, vol. 54, pp. 547-577, 2003.

[47] R. Calvo and S. Kim, "Emotions in text: dimensional and categorical models," Computational Intelligence, 2012.

[48] M. Thelwall, K. Buckley, G. Paltoglou, D. Cai, and A. Kappas, "Sentiment strength detection in short informal text," J. of the American Society for Information Science and Technology, vol. 61, pp. 2544-2558, 2010.

[49] M. Thelwall, K. Buckley, G. Paltoglou, M. Skowron, D. Garcia, S. Gobron, J. Ahn, A. Kappas, D. Küster, and J. A. Holyst, "Damping sentiment analysis in online communication: Discussions, monologs and dialogs," in Computational Linguistics and Intelligent Text Processing, 2013, pp. 1-12.

[50] M. Nguyen, Y. S. Bin, and A. Campbell, "Comparing online and offline self-disclosure: A systematic review," Cyberpsychology, Behavior, and Social Networking, vol. 15, no. 2, pp. 103-111, 2012.

[51] B. Reeves and C. Nass, The media equation: how people treat computers, television, and new media like real people and places. Cambridge University Press, 1996.

[52] J. P. Forgas, “Affective influences on self-disclosure: Mood effects on the intimacy and reciprocity of disclosing personal information," J. of Personality and Social Psychology, vol. 100, no. 3, pp. 449-461, 2011.

[53] W. Hamilton, "The genetical evolution of social behaviour," J. of Theoretical Biology, vol. 7, no. 1, pp. 1-16, 1964.

[54] J. Bates, "The role of emotion is believable agents," Communication of the ACM, vol. 37, no. 7, pp. 122-125, 1994.

[55] J. C. Lester, S. A. Converse, S. E. Kahler, S. T. Barlow, B. A. Stone, and R. S. Bhogal, "The persona effect: Affective impact of animated pedagogical agents," in Proc. CHI97 Conf. on Human factors in computing systems, Los Angeles CA. ACM Press NY USA, 1997, pp. 359-366.

[56] R. Frank, "Introducing moral emotions into models of rational choice," in Feelings and Emotions: The Amsterdam Symposium. Cambridge University Press, 2004, pp. 422-440.

[57] N. Frijda and B. Mesquita, "The social roles and functions of emotions," in Emotion and culture, empirical studies and mutal influence. American Psychological Association, 1994, pp. 51-87.

[58] D. Keltner and J. Haidt, "Social functions of emotions at four levels of analysis," Cognition and Emotion, vol. 13, pp. 505-522, 1999.

[59] M. Skowron and S. Rank, "Affect listeners - from dyads to group interactions with affective dialog systems," in Proc. of AISB/IACAP World Congress 2012, LaCATODA 2012, 2012, pp. 55-60.

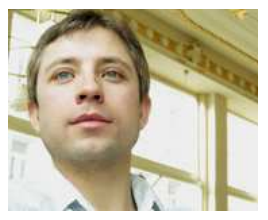

Marcin Skowron is a senior researcher at the Austrian Research Institute for Artificial Intelligence (OFAl). He received an MA degree from the Gdansk University, Poland in 2000, and a PhD degree from Hokkaido University, Japan in 2005. His primary research interests are in cognitive and affective sciences, human-computer interaction, artificial intelligence and Web mining. His recent work is focused around the EU FP7 ICT project CYBEREMOTIONS, where he leads the research related to the development and evaluation of affective human-computer interaction systems, and sentiment-oriented analysis of data on text-based human-computer and human-human online interactions.

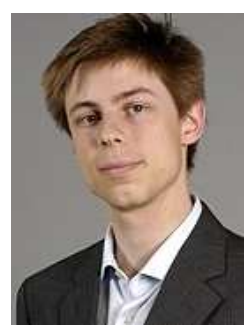

Mathias Theunis is a doctoral candidate in psychology at Jacobs University Bremen, Germany. His research focuses on social processes, emotion, and motivation. As a member of the CYBEREMOTIONS project, he collaborates on the design and evaluation of sentiment mining and Al software.

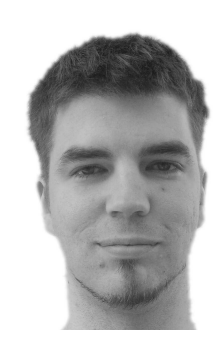

Stefan Rank is a senior researcher at the Austrian Research Institute for Artificial Intelligence (OFAI). He received an MSc and PhD degree from the Vienna University of Technology, Austria, in 2004 and 2009 respectively. His research focuses on computational models of emotion and behavior, their implementation in software agents, and their application in interactive media and agent-based simulation. He was recently involved in the the EU FP7 ICT projects SERA, IRIS and CYBEREMOTIONS, in which his work focused on affective human-computer interaction systems and the application of agent-based modeling in these systems. He is a member of AAAI, and he also serves as portal editor for the HUMAINE association for Affective Computing and Intelligent Interaction.

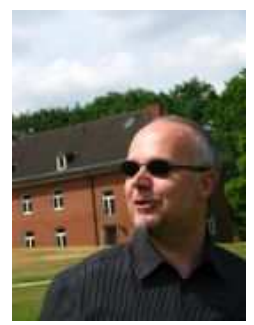

Arvid Kappas is a full professor of psychology at Jacobs University Bremen and has worked and taught in different European countries, Canada, and the United States in the last two decades. His research and teaching focuses on emotion science, communication, and social neuroscience. His laboratory currently investigates psychophysiological and expressive changes as a function of emotional states and social context in Internet interactions. He was an associate editor of Emotion and Biological Psychology and is currently on the editorial board of Cognition and Emotion and the Journal of Nonverbal Behavior. He is also a member of the executive committee of the HUMAINE association. Three major current/recent projects that are funded by the EU and that fall into the domain of Affective Computing are CYBEREMOTIONS, eCUTE, and EMOTE. 